\title{
Dynamic Study of Sodiation Process in Single Crystalline $\alpha-\mathrm{MnO}_{2}$ Nanowires
}

\author{
Yifei Yuan ${ }^{1}$, Anmin $\mathrm{Nie}^{2}$, Wentao Yao ${ }^{2}$, Reza Shahbazian-Yassar ${ }^{2 *}$ \\ 1. Department of Materials Science and Engineering, Michigan Technological University, 1400 \\ Townsend Dive, Houghton, Michigan 49931 USA \\ 2. Department of Mechanical Engineering-Engineering Mechanics, Michigan \\ Technological University, 1400 Townsend Dive, Houghton, Michigan 49931, USA
}

$\alpha-\mathrm{MnO}_{2}$ is widely applied as an energy storage electrode in rechargeable batteries due to its unique $2 \times 2$ tunneled structure that facilitates diffusion of charge carriers ${ }^{[1]}$. By now, it is unclear how the intercalated charge carriers such as $\mathrm{Li}^{+}, \mathrm{Na}^{+}$and $\mathrm{Mg}^{2+}$ interact with the tunnel-based host due to the lack of atomic scale understanding of the tunnel configuration and the complicated effect from generally existing tunnel stabilizers (like $\mathrm{K}^{+}$).

In this paper, using aberration-corrected scanning transmission electron microscopy (ACSTEM) to cross sectioned $\mathrm{K}^{+}$-stabilized $\alpha-\mathrm{MnO}_{2}$ nanowires, the $1 \times 1$ and $2 \times 2$ tunneled structures as well as defective $2 \times 3$ and $2 \times 4$ tunnels are clearly demonstrated at atomic level. An open cell design in TEM for dynamic study of $\alpha-\mathrm{MnO}_{2}$ 's sodiation process confirms that an intermediate phase $\mathrm{Na}_{\mathrm{x}} \mathrm{MnO}_{2}$ will first appear upon sodiation and finally the tunneled structure will totally collapse, generating $\mathrm{Mn}_{2} \mathrm{O}_{3}$ polycrystals embedded in $\mathrm{Na}_{2} \mathrm{O}$ matrix. The originally existing tunnel stabilizer $\mathrm{K}^{+}$will be partially removed upon sodiation, as shown in Figure 1 . It also shows that defective $2 \times 3$ and $2 \times 4$ tunnels function as the fast sodiation path during initial $\mathrm{Na}^{+}$intercalation stage. This study provides fundamental understanding of the tunnel-charge carrier interaction and reveals the structural evolution mechanism of sodiation in $\alpha-\mathrm{MnO}_{2}$. The key role of $2 \times 3$ and $2 \times 4$ tunnels on increasing the discharge rate is also demonstrated, shedding light on potential tunnellevel modification for improving the overall performance of tunnel-based electrodes.

Reference:

[1] Yuliang Cao et al, Advanced Materials 23 (2011), p. 3155 


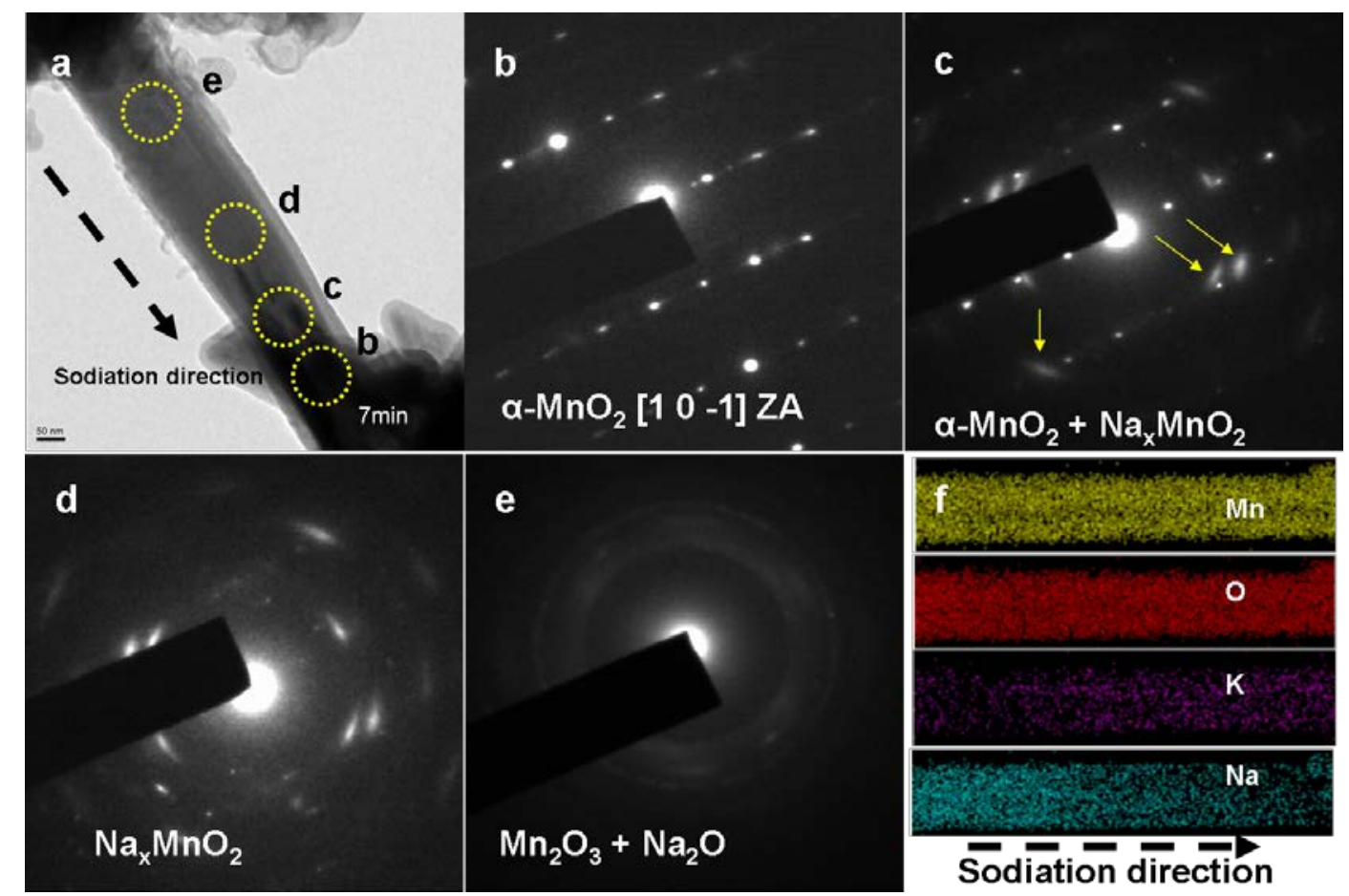

Figure 1 (a) in-situ TEM image of one $\alpha-\mathrm{MnO}_{2}$ nanowire being sodiated with four areas circled as b, c, d and e; (b-e) corresponding selected area diffraction patterns from areas b, c, d and e as indicated in (a); (f) EDS mappings of $\mathrm{Mn}, \mathrm{O}, \mathrm{Na}$ and $\mathrm{K}$ inside one partially sodiated $\mathrm{K}^{+}$stabilized $\alpha-\mathrm{MnO}_{2}$ nanowire. 